Pneumologe 2011 · 8:387-388 DOI 10.1007/s10405-011-0481-y

๑) Springer-Verlag 2011
P. Zabel

Medizinische Klinik Forschungszentrum Borstel/Medizinische Klinik III, UK S-H, Lübeck

\title{
Nichttuberkulöse Mykobakteriosen
}

Ich bin der Bitte des Springer-Verlages, ein Heft über nichttuberkulöse Mykobakteriosen (NTM) zu gestalten, sehr gerne nachgekommen. Da NTM-Infektionen zum ganz überwiegenden Teil die Lunge betreffen, ist insbesondere für Pneumologen das Wissen über diese eher seltenen Erkrankungen von großer Bedeutung. Mein großer Dank gilt daher den Autoren dieses Heftes, die mit ausgewiesener Expertise sich den verschiedenen Aspekten der NTM gewidmet haben, um das derzeitige Wissen über diese Erkrankungen unserer Leserschaft vorzustellen.

Da es bisher weltweit nur wenige epidemiologische Daten zu NTM-Erkrankungen gibt, bin ich Jakko van Ingen (Nijmwegen, NL) und Dirk Wagner (Freiburg) ganz besonders dankbar, dass sie die verfügbaren Erkenntnisse, insbesondere aus Nordamerika und wenigen europäischen Ländern, zusammengetragen und bewertet haben. Die in den Regionen doch sehr unterschiedlichen Inzidenzen und Prävalenzen unterliegen zudem in den letzten Jahren auch noch deutlichen Veränderungen über die Zeit und bezüglich der nachgewiesenen Erregerspezies. Weitere epidemiologische Studien auf diesem Feld sind unabdingbar, um diesen Wandel besser verstehen zu können.

Ein Teil dieser Veränderungen ist sicherlich auch durch zunehmende Fortschritte in der mikrobiologischen Diagnostik von NTM zu finden. Elvira Richter und Doris Hillemann aus dem Nationalen Referenzzentrum für Mykobakterien in Borstel sind besonders ausgewiesen, diese neuesten Nachweismethoden mit genetischer Speziesdifferenzierung zu erläutern und auch die Grenzen der Nachweisbarkeit dieser Erreger (paucibazillär) aus den verschiedenen humanen Untersuchungsmaterialien aufzuzeigen. Die Unterscheidung einer Kolonisation von einer definitiven Infektion ist dabei mikrobiologisch nicht möglich. Es gibt aber Hinweise, dass bereits der alleinige mikrobiologische Nachweis von z. B. M. xenopi im Sinne einer Kolonisation und nicht nur die definitive Erkrankung mit einer ungünstigen Prognose belastet ist [1].

\section{( Kolonisation und Infektion sind mikrobiologisch nicht zu unterscheiden}

NTM-Erkrankungen sind bei einer Vielzahl pulmonaler Krankheiten unbedingt differenzialdiagnostisch zu bedenken, da weder die Klinik noch die radiologischen Veränderungen dieser Erkrankungen spezifisch sind. Ulf Greinert und Werner Lotz aus der Medizinischen Klinik Borstel versuchen als bewährtes Tandem aus erfahrenem Pneumologen und Radiologen, zumindest einige ,spezielle radiologische Befunde" zu definieren, die an eine NTMInfektion denken lassen müssen. Speziesspezifische Befunde sind jedoch praktisch nicht zu definieren, zumal auch die Ausprägung NTM-bedingter radiologischer Veränderungen von der lokalen und systemischen Immunkompetenz des Erkrankten entscheidend mit geprägt wird.

Der Bedeutung der lokalen und systemischen Immundefizienz für das Auftreten und die Ausprägung von NTM-Infektionen wird daher dankenswerter Weise in einem Beitrag von den beiden sehr erfahrenen Infektiologen Jan Rupp (Lübeck) und Bernhard Schaaf (Dortmund) adressiert. Hierin wird deutlich, dass insbesondere die Zunahme aggressiver immun- 
suppressiver Therapiestrategien mit „Biologicals" bei chronisch entzündlichen Erkrankungen häufiger zu NTM-Erkrankungen führt, die nicht nur neu erworben sind, sondern wahrscheinlich auch durch Reaktivierungen entstehen und in Abhängigkeit von der lokalen und systemischen Immunkompetenz (z. B. bei HIV/ Aids) auch einer verlängerten Therapiedauer bedürfen.

\section{( ) Eine Immunsuppression mit „Biologicals" führt häufiger zu NTM-Erkrankungen}

Den Reigen der interessanten Artikel beschließen Christoph Lange und Ulf Greinert aus dem Forschungszentrum Borstel sowie Tom Schaberg aus dem Diakoniekrankenhaus Rotenburg mit dem sehr gelungenen Beitrag zur Therapie der unterschiedlichen NTM-Erkrankungen. In diesem Beitrag wird deutlich, dass bereits die Indikation zur Therapie nur aus der Gesamtschau der klinischen, radiologischen und mikrobiologischen Kriterien gestellt werden kann. Neben nur wenigen allgemeinen Therapieprinzipien, die auch bezüglich einzelner NTM-Spezies in den Empfehlungen der Fachgesellschaften verfügbar sind, ist praktisch in jedem Falle eine individuelle Abwägung der therapeutischer Optionen, zu denen auch die Lungenresektionstherapie gehören kann, vorzunehmen und im Therapieverlauf zu überprüfen.

Ich würde mich sehr freuen, wenn Sie das vorliegende Themenheft von Der Pneumologe über das aktuelle Wissen von NTM-Erkrankungen mit Gewinn für Ihr tägliches ärztliches Handeln lesen, und möchte Sie ausdrücklich ermuntern, bei differenzialdiagnostisch oder therapeutisch schwierigen NTM-Fällen auch zusätzlich Rat und Tat bei erfahrenen Kollegen zu suchen.

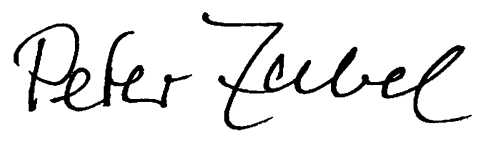

Prof. Dr. P. Zabel

\section{Korrespondenzadresse}

Prof. Dr. P. Zabel

Medizinische Klinik Forschungszentrum Borstel/Medizinische Klinik III, UK S-H, Lübeck Parkallee 35, 23845 Borstel

pzabel@fz-borstel.de

\section{Literatur}

1. Andrejak C, Thomsen VO, Johansen IS et al (2010) Nontuberculous pulmonary mycobacteriosis in Denmark: incidence and prognostic factors. Am J Respir Crit Care Med 181:514-521

\section{DGIM News-App}

\section{für Innere Medizin jetzt im}

\section{iTunes-Store}

iPhone- und iPad-Besitzer können sich ab sofort durch eine kostenlose App schnell und umfassend über Neuigkeiten aus der Inneren Medizin informieren. Im Rahmen der Kooperation zwischen dem Fachinformationsverlag Springer Medizin und der Deutschen Gesellschaft für Innere Medizin wurde dieses mobile Angebot als wichtiger Kommunikationsservice der Gesellschaft für Mitglieder und Interessierte anderer Fachrichtungen entwickelt.

Die App bietet auf übersichtliche Weise informative Inhalte aus Forschung und Praxis mit täglich aktualisierten Meldungen aus der Inneren Medizin, Kongressnachrichten und Neuigkeiten aus den Bereichen Praxismanagement, Wirtschaft und Gesundheitspolitik. Über die App sind zudem alle aktuellen Meldungen der DGIM aufrufbar und es besteht die Möglichkeit, direkt per E-Mail Kontakt mit der Gesellschaft aufzunehmen.

Die Nutzer können mit der Applikation bequem Artikel in einer Merkliste ablegen, sie außerdem bei Facebook und Twitter posten und Artikelempfehlungen per E-Mail an Kollegen weiterleiten.

Jeder, der bereits auf springermedizin.de registriert ist, kann mit der Eingabe seines Passworts sofort auf alle Informationen zugreifen. Nutzer, die sich bisher noch nicht angemeldet haben, können dies mit einer kostenlosen Kurzregistrierung innerhalb der App nachholen. Als besonderen Service erhalten registrierte Nutzer eine Benachrichtigung, wenn die neue Ausgabe der Fachzeitschrift Der Internist erscheint. In dieser Meldung ist bereits das komplette Inhaltsverzeichnis der betreffenden Ausgabe enthalten. So behalten Sie die aktuellen Themen immer im Blick. Die App stellt relevante Inhalte aus dem Praxisoder Klinikalltag dar und ist kostenlos über den iTunes-Store erhältlich.

Springer Medizin hat in diesem Jahr bereits zwei Apps (News-App Kardiologie und Ärzte Zeitung) im iTunes-Store veröffentlicht, eine weitere von der österreichischen Ärzte Woche erscheint im September. 